ROME1-1206/98

TUM-HEP-312/98

\title{
MODEL INDEPENDENT DETERMINATION OF THE LIGHT-CONE WAVE FUNCTIONS FOR EXCLUSIVE PROCESSES
}

\author{
U. Aglietti ${ }^{1}$, M. Ciuchini ${ }^{2}$, G. Corbò ${ }^{1}$, \\ E. Franco ${ }^{1}$, G. Martinelli ${ }^{1}$, L. Silvestrini ${ }^{3}$ \\ 1 Dipartimento di Fisica, Università di Roma "La Sapienza" and INFN, \\ Sezione di Roma, P.le A. Moro 2, I-00185 Rome, Italy. \\ ${ }^{2}$ Dipartimento di Fisica, Università di Roma Tre and INFN, \\ Sezione di Roma Tre, Via della Vasca Navale 84, I-00146 Rome, Italy. \\ ${ }^{3}$ Physik Department, Technische Universität München, \\ D-85748 Garching, Germany.
}

\begin{abstract}
We present a method to compute, by numerical simulations of lattice QCD, the light-cone wave functions which enter exclusive processes at large momentum transfer, such as electromagnetic elastic scatterings, or exclusive semi-leptonic decays as $B \rightarrow \pi$ $(B \rightarrow \rho)$ and radiative decays as $B \rightarrow K^{*} \gamma$. The method is based on first principles and does not require any model assumption.
\end{abstract}


In this paper we propose a method to compute, by numerical simulations on the lattice, the light-cone wave functions which allow to predict the form factors relevant in many exclusive processes, such as electromagnetic elastic scattering at large momentum transfer, or exclusive semi-leptonic decays as $B \rightarrow \pi(B \rightarrow \rho)$ and $B \rightarrow K^{*} \gamma$ decays [1]-[5]. Our approach allows to compute the form factors in exclusive semileptonic $B$ decays at small values of the invariant mass of the lepton pair, $q^{2}$, a region which is not accessible by standard lattice techniques, which are confined in the region $q^{2} \sim q_{\max }^{2}$ [6]-11]. We also show that, from suitable combinations of lattice correlation functions, it is possible to determine the full light-cone wave function, denoted generically as $\Phi$, and not only its moments, as done in the past [12]. The possibility of computing directly $\Phi$ is a significative advantage: higher moments of the light-cone wave functions, being related to higher dimension operators, are in general afflicted by power divergencies when a hard cutoff 1 is used (or renormalon ambiguities in dimensional regularizations). This makes very problematic the definition of the renormalized operators, i.e. those which have finite matrix elements when the cutoff is removed 13. With our method, instead, no renormalization is needed as we get directly $\Phi$ from the appropriate correlation functions. The technique described below strictly follows a similar proposal recently made to determine the shape function for inclusive heavy-hadron decays [14].

We now explain how the method works. The light-cone wave functions are universal quantities, i.e. quantities independent from the process at hand (electromagnetic elastic scattering at large momentum transfer, exclusive $B$ decays, etc.). Thus, in order to illustrate our proposal, we start by considering a very simple prototype which enters, for example, the elastic $\gamma^{*}(q)+\pi \rightarrow \pi$ scattering [1] and $B \rightarrow \pi$ semileptonic decays [2]

$$
F^{\mu}\left(\vec{p}_{\pi}, q\right) \equiv i \int d^{4} x e^{i q \cdot x}\left\langle\pi^{-}\left(\vec{p}_{\pi}\right)\left|\bar{d}(x) \gamma^{\mu} \gamma_{5} S(x ; 0) u(0)\right| 0\right\rangle
$$

where, for simplicity, and without loss of generality, we have taken $S(x ; 0)$ to be the scalar Feynman propagator, satisfying the differential equation

$$
-D^{2} S(x ; 0)=\delta^{4}(x-0)
$$

With this choice the multilocal operator appearing in eq. (1) is gauge-invariant.

$F^{\mu}\left(\vec{p}_{\pi}, q\right)$ can be written as

$$
F^{\mu}\left(\vec{p}_{\pi}, q\right)=i \int d^{4} x\left\langle\pi^{-}\left(\vec{p}_{\pi}\right)\left|\bar{d}(x) \gamma^{\mu} \gamma_{5} S_{Q}(x ; 0) u(0)\right| 0\right\rangle,
$$

\footnotetext{
${ }^{1}$ In the lattice regularization the hard cutoff is given by the inverse lattice spacing $1 / a$.
} 
where $S_{Q}(x ; 0) \equiv e^{i q \cdot x} S(x ; 0)$. For simplicity we consider first the case of a pion with a small momentum (at rest), i.e. $\left|\vec{p}_{\pi}\right| \ll|\vec{q}|\left(\vec{p}_{\pi}=0\right)$. For $-q^{2}=Q^{2} \gg p_{\pi}^{2}=M_{\pi}^{2}$, we can separate the large frequency modes $\left(\sim Q=\sqrt{Q^{2}}\right)$ from the low energy modes $\left(\sim \Lambda_{Q C D}\right)$ and expand $S_{Q}(x ; 0)$ in powers of $\Lambda_{Q C D} / Q$

$$
S_{Q}(x ; 0) \simeq\left(\frac{1}{-Q^{2}+2 i q \cdot D+i \epsilon}\right)_{(x ; 0)} .
$$

In eq. (14) we kept only the leading terms of the expansion in powers of $1 / Q$, and those which become leading when $u=Q^{2} /\left(2 p_{\pi} \cdot q\right) \sim 1$. Using eq. (4) one finds

$$
F^{\mu}\left(\vec{p}_{\pi}, q\right)=f_{\pi} p_{\pi}^{\mu} \int_{0}^{1} d u \frac{\Phi_{\pi}(u)}{-Q^{2}+2 u q \cdot p_{\pi}+i \epsilon}
$$

where the light-cone wave function $\Phi_{\pi}(u)$ is defined through the relations

$$
\begin{aligned}
\left\langle\pi^{-}\left(\vec{p}_{\pi}\right)\left|\bar{d}(0) \gamma^{\mu} \gamma_{5}\left(i D^{\mu_{1}}\right) \ldots\left(i D^{\mu_{n}}\right) u(0)\right| 0\right\rangle & =-i f_{\pi} \mathcal{M}_{n} p_{\pi}^{\mu} p_{\pi}^{\mu_{1}} \ldots p_{\pi}^{\mu_{n}} \\
& +i \mathcal{B}_{n} \delta^{\mu \mu_{1}} p_{\pi}^{\mu_{2}} \ldots p_{\pi}^{\mu_{n}}+\ldots
\end{aligned}
$$

with

$$
\mathcal{M}_{n}=\int_{0}^{1} d u u^{n} \Phi_{\pi}(u)
$$

In eq. (6), the contributions proportional to $\mathcal{B}_{n}$ are suppressed by higher powers of $1 / Q$ in all the relevant kinematical region.

We now consider the Fourier transform of $F^{\mu}\left(\vec{p}_{\pi}, q\right)$ defined as

$$
F^{\mu}\left(t, \vec{p}_{\pi}, \vec{q}\right)=\int \frac{d q_{0}}{2 \pi} e^{-i q_{0} t} F^{\mu}\left(\vec{p}_{\pi}, q\right)
$$

For $t \geq 0$, by closing the contour of integration over $q_{0}$ below the real axis, we find

$$
F^{\mu}\left(t, \vec{p}_{\pi}, \vec{q}\right)=-i f_{\pi} p_{\pi}^{\mu} \int_{0}^{1} d u \Phi_{\pi}(u) \frac{e^{-i\left(-u E_{\pi}+\sqrt{\vec{q}^{2}+u^{2} E_{\pi}^{2}+2 u \vec{q} \cdot \vec{p}_{\pi}}\right) t}}{2 \sqrt{\vec{q}^{2}+u^{2} E_{\pi}^{2}+2 u \vec{q} \cdot \vec{p}_{\pi}}}
$$

If, for consistency with the order at which we are working, we neglect the terms of $\mathcal{O}\left(M_{\pi}^{2} / Q^{2}\right)$ and $\mathcal{O}\left(\vec{p}_{\pi}^{2} / \vec{q}^{2}\right)$, we get

$$
F^{\mu}\left(t, \vec{p}_{\pi}, \vec{q}\right) \simeq-i f_{\pi} p_{\pi}^{\mu} \int_{0}^{1} d u \Phi_{\pi}(u) \frac{e^{-i\left(-u E_{\pi}+\sqrt{\vec{q}^{2}+2 u \vec{q} \cdot \vec{p}_{\pi}}\right) t}}{2 \sqrt{\vec{q}^{2}+2 u \vec{q} \cdot \vec{p}_{\pi}}} .
$$

It is convenient to write eq. (9) as follows

$$
F^{\mu}\left(t, \vec{p}_{\pi}, \vec{q}\right)=-i f_{\pi} p_{\pi}^{\mu} \int_{0}^{1} d u \Phi_{\pi}(u) \frac{e^{-i u\left(-E_{\pi}\left(\vec{p}_{\pi}\right)+E_{\pi}\left(\vec{p}_{\pi}+\vec{q} / u\right)\right) t}}{2 u E_{\pi}\left(\vec{p}_{\pi}+\vec{q} / u\right)},
$$


where $E_{\pi}(\vec{p})=\sqrt{\vec{p}^{2}+M_{\pi}^{2}}$. In the latter form, one recognizes that eq. (9) is valid for arbitrary pion momenta. In the general case, eqs. (8)-(11) can be derived from the following expression

$$
F^{\mu}\left(\vec{p}_{\pi}, q\right)=f_{\pi} p_{\pi}^{\mu} \int_{0}^{1} d u \frac{\Phi_{\pi}(u)}{W^{2}+2\left(q+p_{\pi}\right) \cdot k+i \epsilon},
$$

where $W^{2}=\left(q+p_{\pi}\right)^{2}=Q^{2}(1-x) / x$ is the squared invariant mass of the recoiling hadron system and $k=u p_{\pi}-p_{\pi}$ is the residual momentum of the struck parton $\left(\left\langle D^{\mu_{1}} \ldots D^{\mu_{n}}\right\rangle \sim k^{\mu_{1}} \ldots k^{\mu_{n}}=\mathcal{O}\left(\Lambda_{Q C D}^{n}\right)\right)$. The expression above shows that different dynamics which occur depending on the value of $x$. At small $x$, we can retain only the $W^{2}$ term in the denominator and the amplitude becomes proportional to the lowest moment of $\Phi_{\pi}$ which is unity; when $W^{2} \sim Q \Lambda_{Q C D}$, all the moments become comparable and we need the full wave function $\Phi_{\pi}$; finally in the region where $W^{2} \sim \Lambda_{Q C D}^{2}$, the light-cone approach fails. This is the analog of what happens in inclusive decays at the end point of the energy spectrum [14].

It is straightforward to make the analytic continuation of the above expression to the Euclidean space-time which is used in numerical simulations

$$
\begin{aligned}
F^{\mu}\left(t, \vec{p}_{\pi}, \vec{q}\right) & \equiv-i f_{\pi} p_{\pi}^{\mu} F\left(t, \vec{p}_{\pi}, \vec{q}\right) \\
& =-i f_{\pi} p_{\pi}^{\mu} \int_{0}^{1} d u \Phi_{\pi}(u) \frac{e^{-u\left(-E_{\pi}\left(\vec{p}_{\pi}\right)+E_{\pi}\left(\vec{p}_{\pi}+\vec{q} / u\right)\right) t}}{2 u E_{\pi}\left(\vec{p}_{\pi}+\vec{q} / u\right)}
\end{aligned}
$$

By studying the time-dependence of $F^{\mu}\left(t, \vec{p}_{\pi}, \vec{q}\right)$ at several values of $\vec{q}$ and $\vec{p}_{\pi}$, we can unfold the integral above and and extract the light-cone wave function $\Phi_{\pi}$.

We give below the practical recipe to implement the calculation of $F^{\mu}\left(t, \vec{p}_{\pi}, \vec{q}\right)$ in lattice simulations. From the definition of $F^{\mu}\left(\vec{p}_{\pi}, q\right)$ in eq. (11), one has

$$
F^{\mu}\left(t, \vec{p}_{\pi}, \vec{q}\right)=i \int d^{3} x e^{-i \vec{q} \cdot \vec{x}}\left\langle\pi\left(\vec{p}_{\pi}\right)\left|\bar{d}(\vec{x}, t) \gamma^{\mu} \gamma_{5} S(\vec{x}, t ; \overrightarrow{0}, 0) u(0)\right| 0\right\rangle .
$$

The above amplitude can be extracted directly from a suitable ratio of lattice threeand two-point correlation functions

$$
R\left(t, \vec{p}_{\pi}, \vec{q}\right)=\lim _{t_{f} \rightarrow \infty} e^{-E_{\pi} t} \frac{G_{3}^{\mu}\left(t_{f}, t, \vec{p}_{\pi}, \vec{q}\right)}{G_{2}^{\mu}\left(t_{f}, \vec{p}_{\pi}\right)}
$$

where $E_{\pi}=\sqrt{M_{\pi}^{2}+\vec{p}_{\pi}^{2}}$;

$$
G_{3}^{\mu}\left(t_{f}, t, \vec{p}_{\pi}, \vec{q}\right)=\int d^{3} x e^{i \vec{q} \cdot \vec{x}}\left\langle 0\left|\Pi_{\vec{p}_{\pi}}\left(t_{f}\right) \bar{d}(\vec{x}, t) \gamma^{\mu} \gamma_{5} S(\vec{x}, t ; \overrightarrow{0}, 0) u(0)\right| 0\right\rangle
$$

and

$$
G_{2}^{\mu}\left(t_{f}, \vec{p}_{\pi}\right)=\left\langle 0\left|\Pi_{\vec{p}_{\pi}}\left(t_{f}\right) A_{\vec{p}_{\pi}}^{\mu \dagger}(0)\right| 0\right\rangle
$$


$\Pi_{\vec{p}_{\pi}}(t)$ and $A_{\vec{p}_{\pi}}^{\mu \dagger}(t)$ are the pion interpolating field and the axial current $\left(A^{\mu}(x)=\right.$ $\left.\bar{u}(x) \gamma^{\mu} \gamma_{5} d(x)\right)$ with definite spatial momentum $\vec{p}_{\pi}$

$$
\Pi_{\vec{p}_{\pi}}(t)=\int d^{3} x e^{i \vec{p}_{\pi} \cdot \vec{x}} \partial_{\mu} A^{\mu}(\vec{x}, t), \quad A_{\vec{p}_{\pi}}^{\mu}(t)=\int d^{3} x e^{i \vec{p}_{\pi} \cdot \vec{x}} A^{\mu}(\vec{x}, t) .
$$

In the Euclidean, using the transfer matrix formalism, we have

$$
\Pi_{\vec{p}_{\pi}}(\vec{x}, t)=e^{\hat{H} t} \Pi_{\vec{p}_{\pi}}(\vec{x}) e^{-\hat{H} t},
$$

so that the correlation functions have an exponential dependence on the energy of the external states. This implies that, in the limit $t_{f} \rightarrow \infty$, the lightest state, corresponding to a pion, dominates the correlation functions (16) and (17), since all higher-energy states are exponentially suppressed. In this limit, with $t>0$, we then obtain

$$
R\left(t, \vec{p}_{\pi}, \vec{q}\right) \rightarrow F\left(t, \vec{p}_{\pi}, \vec{q}\right)
$$

which is the desired quantity.

It is obvious that the same technique can be used to compute all the light-cone functions which have been introduced in the literature. In the case of the pion, for example, those denoted as $\Phi_{p}$ and $\Phi_{\sigma}$ in ref. [2] correspond to the non-local amplitude of eq. (1), where $\gamma^{\mu} \gamma_{5}$ is replaced by $i \gamma_{5}$ and $\sigma_{\mu \nu}$ respectively. The same holds true, taking into account all the complications entailed by the polarization of the physical states, in the case of the $\Phi_{\mathrm{S}}$ which are relevant for the $\rho$ [5], the $K^{*}$ meson [4] and the nucleon [3] cases.

In practical calculations one may use either the scalar propagator defined in eq. (2) or the approximate propagator introduced in eq. (四). In the general case, the latter can be written as

$$
S_{Q}(x ; 0)=\left(\frac{1}{\left(q+p_{\pi}\right)^{2}+2 i\left(q+p_{\pi}\right) \cdot D+i \epsilon}\right)_{(x ; 0)} .
$$

$S_{Q}(x)$ can be written as

$$
S_{Q}(x)=\frac{e^{+i Q x_{+} / 2}}{Q} S_{L E E T}(x)
$$

where $x_{+}=n \cdot x$, with $n^{\mu}=\left(q^{\mu}+p_{\pi}^{\mu}\right) / Q$, and $S_{L E E T}(x)$ is the light-cone propagator of the Large Energy Effective Theory (LEET) [15], which satisfies the equation

$$
2 i D_{+} S_{L E E T}(x)=\delta^{4}(x)
$$

$n^{\mu}$ is the appropriate vector which becomes light-like in the elastic region, $n^{2}=$ $W^{2} / Q^{2} \sim \Lambda_{Q C D} / Q$ when $W \sim Q \Lambda_{Q C D}$. Thus, the extraction of the $\Phi$ s from $S_{Q}(x)$ is equivalent to the use of the LEET. Note that the calculation of the physical light-cone 
function $\Phi_{\pi}$ using the full propagator of eq. (2) does not need any renormalization (for $\Phi_{p}$ and $\Phi_{\sigma}$, the same renormalization constants of the operators $\bar{\psi} \gamma_{5} \psi$ and $\bar{\psi} \sigma^{\mu \nu} \psi$ must be applied). The same calculation using the $S_{L E E T}$ requires, instead an overall (further) logarithmic renormalization of the amplitude [16], which can be computed in lattice perturbation theory. The ultraviolet divergences of the LEET correspond perturbatively to infrared divergences in the full theory [17]. In the latter case the infrared divergences are automatically regularized by the non-perturbative contributions in the physical matrix elements and no renormalization is required.

As discussed in ref. [14], it is not clear whether the use of $S_{L E E T}(x)$ will be convenient in practice, since this propagator is much more singular than the full one. For this reason we expect that the correlation functions computed in numerical simulations using $S_{L E E T}(x)$ will be affected by larger statistical fluctuations.

\section{Acknowlegments}

L.S. acknowledges the support of German Bundesministerium für Bildung und Forschung under contract 06 TM 874 and DFG Project Li 519/2-2. We acknowledge partial support by M.U.R.S.T.

\section{References}

[1] I.I. Balitsky, V.M. Braun, A.V. Kolesnichenko, Nucl. Phys. B 312 (1989) 509; V.M. Braun, I.B. Filyanov, Z. Phys. C 44 (1989) 157; ibid. 48 (1990) 239; V.L. Chernyak, I.R. Zhitnitsky, Nucl. Phys. B345 (1990) 137; P. Ball, V.M. Braun, H.G. Dosch, Phys. Rev. D 44 (1991) 3567.

[2] V.M. Belyaev, A. Khodjamirian, R. Rückl, Z. Phys. C60 (1993) 349; A. Khodjamirian and R. Rückl WUE-ITP-97-049, to appear in Heavy Flavors, 2nd edition, eds., A.J. Buras and M. Linder (World Scientific), hep-ph/9801443.

[3] V.L. Chernyak, A.R. Zhitntsky, Phys. Rep. 112 (1984) 173.

[4] A. Ali, V.M. Braun and H. Simma, Z. Phys. C63 (1994) 437.

[5] P. Ball and V.M. Braun, Phys. Rev. D54 (1996) 2182; P. Ball, V.M. Braun, Y. Koike, K. Tanaka, hep-ph/9802299.

[6] T. Bhattacharya and R. Gupta, Proc. Lattice 94, 12th Int. Symp. on Lattice Field Theory, Bielefeld, Germany, 1994, Nucl. Phys. B (Proc. Suppl.) 42 (1995) 935 and 47 (1996) 481 
[7] APE Collaboration, C.R. Allton et al., Phys. Lett. B 345 (1995) 513

[8] As. Abada et al., Nucl. Phys. B 416 (1994) 675

[9] UKQCD Collaboration, D.R. Burford et al., Nucl. Phys. B 447 (1995) 425

[10] UKQCD Collaboration, J.M. Flynn et al., Nucl. Phys. B 461 (1996) 327

[11] UKQCD Collaboration, L. Del Debbio et al., Granada-Marseille-Southampton preprint, UG-DFM-4/97, CPT-97/P.3505, SHEP-97-13, hep-lat/9708008.

[12] G. Martinelli and C.T. Sachrajda, Phys. Lett. 190B (1987) 15 and Nucl. Phys. B316 (1989) 305.

[13] G. Martinelli and C.T. Sachrajda, Phys. Lett. B354 (1995) 423 and Nucl. Phys. B478 (1996) 660.

[14] U. Aglietti et al., ROME1-1204/98, hep-ph/9804416.

[15] M.J. Dugan and B. Grinstein, Phys. Lett. B255 (1991) 583.

[16] G.P. Korchemsky and G. Sterman, Phys. Lett. B340 (1994) 96; A.G. Grozin and G.P. Korchemsky, Phys. Rev. D53 (1996) 1378.

[17] U. Aglietti, G. Corbò and L. Trentadue, hep-ph/9712237. 